Research Article

\title{
Hepatitis C Virus Core Protein Enhances Natural Killer Cell Depletion of Plasmacytoid Dendritic Cells
}

\author{
Janelle Guy $^{1 \Perp}$, Emmanuella Sobo ${ }^{2}$, Lechi Nwanegwo ${ }^{2}$, Kadir Aslan ${ }^{1 \Perp}$ \\ ${ }^{1}$ Department of Civil Engineering, Morgan State University, 1700 East Cold Spring Lane, Baltimore, MD 21251 USA. \\ ${ }^{2}$ Department of Biology, Morgan State University, USA. \\ C Corresponding authors. E-mail: jaguy9@morgan.edu; kadir.aslan@morgan.edu
}

Received: Jun. I, 2018; Accepted: Feb. 5, 2019; Published: Mar. I, 2019.

Citation: Janelle Guy, Emmanuella Sobo, Lechi Nwanegwo, and Kadir Aslan, Hepatitis C Virus Core Protein Enhances Natural Killer Cell Depletion of Plasmacytoid Dendritic Cells. Nano Biomed. Eng., 2019, I I (I): 44-57.

DOI: 10.5101/nbe.v11i1.p44-57.

\begin{abstract}
Investigation of the bi-directional relationship between natural killer (NK) cells and plasmacytoid dendritic cells (pDCs) is critical in understanding antiviral immunity. In the present study, we determined whether the tumor necrosis factor apoptosis-inducing ligand (TRAIL) pathway was responsible for increased apoptosis of pDCs in hepatitis C virus (HCV) infection. We stimulated peripheral blood mononuclear cells (PBMCs) with recombinant HCV core protein within 12 hours to measure the relative expression of tumor necrosis factor apoptosis-inducing receptor 1 (TRAIL-R1) and TRAIL-R2 in pDCs using flow cytometry and image cytometry. We also measured the relative expression of TRAIL in NK cells after stimulation with recombinant HCV core protein using flow cytometry and image cytometry. Using flow cytometry, our results show that within 12 hours of stimulation, HCV core protein increases TRAIL-R1 on pDCs by $0.01 \%$, CD56 expression by $0.66 \%$, and TRAIL expression by $0.66 \%$, in NK cells as compared to unstimulated PBMCs. ELISA and fluorescence spectroscopy results showed that HCV core protein decreases Bcl-2 expression in PBMCs and in pDCs by $36 \%$ and 3\%, respectively. Our results suggest that HCV core protein increases NK cell deletion of pDCs, independent of the Bcl-2 pathway, contributing to HCV viral escape from immune responses, which may result in chronic HCV infection.
\end{abstract}

Keywords: Hepatitis; Innate immunity; Host response; Apoptosis; Viral pathogenesis

\section{Introduction}

The type of adaptive immune response against hepatitis $\mathrm{C}$ virus (HCV) infection greatly depends on the effectiveness of the innate immune response. Two important cell types that help mediate adaptive immunity include dendritic cells (DC) and natural killer (NK) cells. There are two major subtypes of DCs, which include myeloid DCs and plasmacytoid dendritic cells (pDCs). Although the population of pDCs makes up a small percentage ( $0.1 \%-0.3 \%)$ of the total peripheral blood mononuclear cell (PBMC) population, pDCs are the major producers of type I interferon (IFN), which is critical to reducing viral replication within the infected host.

It is known that HCV infection mainly targets hepatocytes, yet the number and function of pDCs in chronically HCV-infected individuals is decreased [1-3]. Some reports have suggested that the reduced number of pDC in chronic HCV infection is due to increased homing of pDC to the liver $[1,4-6]$. However, few reports have investigated if the number 
of pDCs is related to apoptotic events occurring because of HCV pathogenesis [2, 5]. These reports suggest that pDC apoptosis is mainly due to monocytederived TNF- $\alpha$ or IL-10 [5].

Moreover, the bi-directional communication between NK cells and pDCs is very important for both NK cell activation and pDC maturation and function $[7,8]$. pDCs require cell-cell contact with NK cells for maturation $[7,9]$. Through this interaction, pDCs increase proliferation of NK cells as well as increase the activity of NK cells [10]. Moreover, a key trait of NK cells is their ability to survey tissues and blood cells for the expression of self-antigens, which include the major histocompatibility complex (MHC) [10]. Particularly, NK cells scan cells for upregulation of MHC type I expression [11-13]. NK cells can differentiate tumor or infected cells due to the downregulation of MHC class I on these cells [10, 12, 13]. When this recognition occurs, NK cells are activated and begin the process of rapidly eliminating these cells by the secretion of cytokines or chemokines [14]. NK cells must also maintain communication with DCs because immature DCs are able to send out abortive and tolerogenic signals to $\mathrm{T}$ cells, which can hinder adaptive immunity $[10,15,16]$.

To add, containment of infection is modulated by pDC editing or killing by NK cells. Mature DCs have upregulated MHC class I and express co-stimulatory molecules that allow DCs the ability to migrate to inductive sites [7,9]. NK cells regulate DC exposure to antigens by controlling the DC ratio in inductive sites. For example, when low levels of NK cells are present, DCs are induced to mature; however, when there are high levels of NK cells, DCs are eliminated by NK cells through an HLA-E-dependent mechanism $[7,9,16]$. Thus, editing of DCs regulated by this NK: DC ratio maintains homeostasis. However, it remains unclear how HCV alters NK cell editing of DCs.

The aim of the present study is to investigate whether enhanced cytotoxicity of NK cells contributes to cell-mediated apoptosis of pDCs in the presence of HCV via the TRAIL-mediated pathway. We hypothesized that reduced $\mathrm{pDC}$ frequency is caused by increased NK cell depletion of pDC apoptosis in HCV infection, providing a mechanism of escape for $\mathrm{HCV}$ from host antiviral strategies.

\section{Experimental}

\section{Peripheral blood mononuclear cells}

Peripheral blood mononuclear cells (PBMCs) were purchased from Interstate Blood Bank (Memphis, TN). PBMCs obtained from Interstate Blood Bank were collected in acid-citrate dextrose (ACD) from healthy donors negative for hepatitis C virus (HCV), hepatitis B virus (HBV), and human immunodeficiency virus (HIV). PBMCs were cryopreserved in liquid nitrogen for 6 to 14 days before thawing. Upon removal from cryopreservation, PBMCs were rapidly thawed in a $37{ }^{\circ} \mathrm{C}$ water bath, approximately $3 \mathrm{~min}$. While on ice, PBMCs were transferred to a $50 \mathrm{~mL}$ centrifuge tube. The cryovial was rinsed with $1 \mathrm{~mL}$ of thawing media (1X Hank's Balanced Salt Solution [HBSS], supplemented with $10 \%$ FBS), and the remaining contents in the cryovial were transferred to the centrifuge tube. Ten milliliters thawing media was added to the $50 \mathrm{~mL}$ centrifuge tube to resuspend PBMCs, and an aliquot was removed to determine cell viability. The PBMCs were centrifuged at $500 \mathrm{x} g$ for $12 \mathrm{~min}$ at $4{ }^{\circ} \mathrm{C}$, and the thawing media was discarded from the cell pellet. The cell pellet was washed again with $10 \mathrm{~mL}$ complete media (RPMI 1640, supplemented with $10 \%$ FBS) and centrifuged at 500 $\mathrm{x} g$ for $12 \mathrm{~min}$ at $4{ }^{\circ} \mathrm{C}$. The PBMCs were re-suspended in $10 \mathrm{~mL}$ complete media. Thereafter, PBMCs were transferred to a T-25 $\mathrm{cm}^{2}$ flask for overnight recovery at $37{ }^{\circ} \mathrm{C}$ with $5 \% \mathrm{CO}_{2}$ until cell stimulation experiments.

\section{PBMC stimulation}

After overnight recovery, cell viability was determined for recovered PBMCs using the trypan blue exclusion assay. The cells were seeded in 24-well or 96-well cell culture plates at a density of $2.0 \times 10^{5}$ cells/ $\mathrm{mL}$ in complete media. LPS from Salmonella enterica serotype Minnesota (Sigma Aldrich) or recombinant HCV core protein (Abcam) was added to PBMCs at a final concentration of $10 \mu \mathrm{g} / \mathrm{mL}$. PBMCs were incubated with antigens for either three, six, or twelve hours of incubation. We chose these incubation periods because of the short life-span of pDCs. It has been shown that an insignificant amount of pDCs survived after 2 days of culture [17].

\section{Flow cytometric analysis of TRAIL, TRAIL-R1, and TRAIL-R2}

After stimulation, PBMCs were harvested by centrifugation at $500 \mathrm{xg}$ for $12 \mathrm{~min}$ at $4{ }^{\circ} \mathrm{C}$. PBMCs were washed once with phosphate buffered saline (PBS), and centrifuged at $500 \mathrm{xg}$ for $12 \mathrm{~min}$ at $4{ }^{\circ} \mathrm{C}$. The cell pellet was re-suspended in FACS Staining Buffer (PBS, supplemented with 10\% FBS), and washed twice before surface staining. PBMCs were 
surface-stained in $5 \mathrm{~mL}$ FACS tubes with PE-labeled TRAIL, TRAIL-R1, or TRAIL-R2, FITC-labeled BDCA2 and APC-labeled BDCA4, or APC-labeled CD56 monoclonal antibodies (BioLegend, San Diego, CA) at room temperature in the dark for $30 \mathrm{~min}$. After surface-staining, cells were washed extensively with FACS Staining Buffer before overnight fixation in $0.1 \%$ paraformaldehyde (PFA) in PBS. Cells were acquired with a BDFACS Canto II, and data were analyzed using the FlowJo software. A sample gating strategy for isolation of cells is shown in Fig. 1.

\section{Image cytometry analysis of TRAIL, TRAIL-R1, and TRAIL-R2}

After stimulation, PBMCs were harvested by centrifugation at $500 \times \mathrm{g}$ for $12 \mathrm{~min}$ at $4{ }^{\circ} \mathrm{C}$. PBMCs were washed once with PBS, and centrifuged at $500 \mathrm{x} g$ for $12 \mathrm{~min}$ at $4{ }^{\circ} \mathrm{C}$. The cell pellet was re-suspended in FACS staining buffer and washed twice before surface staining. PBMCs were surface-stained in 96-well flat bottom plates with PE-labeled TRAIL, TRAIL-R1, and TRAIL-R2, FITC-labeled BDCA2 and APC-labeled BDCA4, or APC-labeled CD56 monoclonal antibodies overnight in the dark at $4{ }^{\circ} \mathrm{C}$. After surface-staining, cells were washed several times with FACS Staining Buffer before overnight fixation in 0.1\% PFA in PBS. Cells were acquired on an Amnis Flowsight Imaging Flow Cytometer and analyzed using Ideas, version 6.2. The experimental design for flow cytometry and image cytometry analysis is displayed in Fig. 2.

\section{Determination of caspase-3 and Bcl-2 levels in PBMCs exposed to HCV core protein using an indirect ELISA}

After stimulation, PBMCs were harvested by centrifugation at $500 \times \mathrm{g}$ for $8 \mathrm{~min}$ at $4{ }^{\circ} \mathrm{C}$. PBMC cell pellets were washed once with ice-cold PBS and centrifuged at $500 \mathrm{xg}$ for $8 \mathrm{~min}$ at $4{ }^{\circ} \mathrm{C}$. The PBS was discarded, and the PBMCs were re-suspended in $100 \mu \mathrm{L}$ of NETN lysis buffer. Samples were agitated on ice for $30 \mathrm{~min}$ and centrifuged at 12,000 rpm for $20 \mathrm{~min}$. The supernatant was removed and placed into a new microcentrifuge tube for storage at $-80{ }^{\circ} \mathrm{C}$ until analysis. We used the Bradford assay to quantify protein concentration in whole cell lysates.

We diluted whole cell lysates to $20 \mu \mathrm{g} / \mathrm{mL}$. Fifty microliters of diluted sample were used to coat the wells of a 96 well ELISA microplate for 2 hours at

Sample gating strategy for multicolor FACS
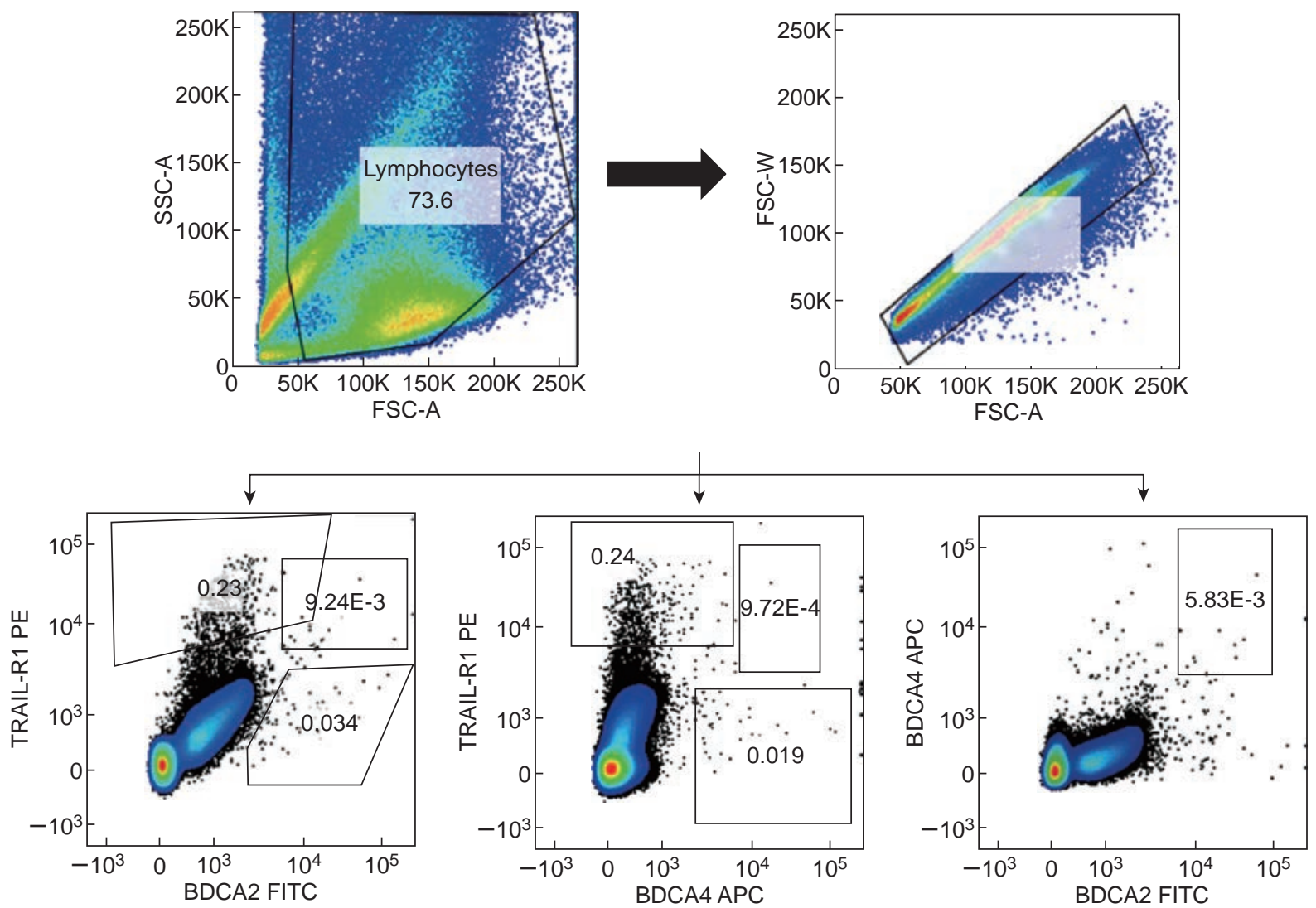

Fig. 1 Sample gating strategy for multi-color FACS. 
Detection of TRAIL and death receptors in NK cells and pDCs

NK cells

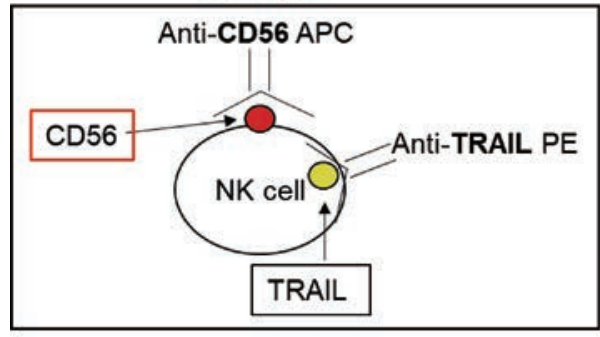

pDCs

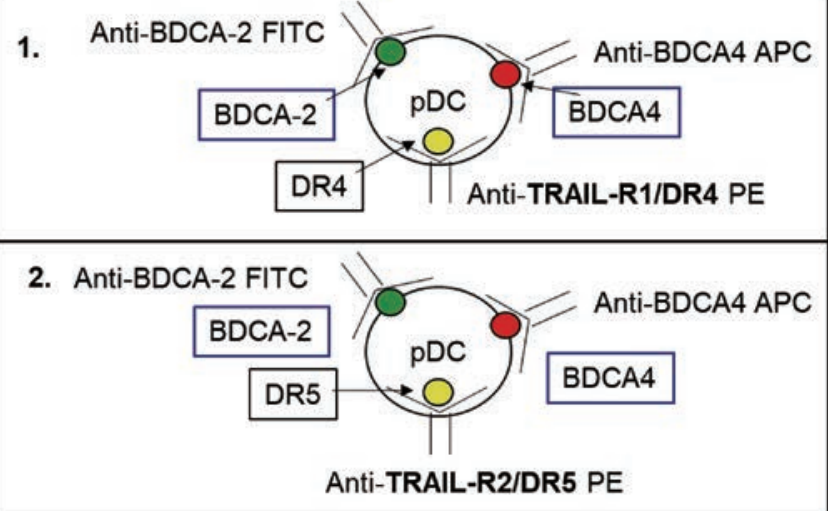

Fig. 2 Experimental design for FACS detection of TRAIL in NK cells and TRAIL-R1 and TRAIL-R2 in pDCs.

room temperature. We removed the samples from the wells and blocked the coated wells with blocking buffer (1\% BSA in PBS for 1 hour at room temperature. Sample wells were washed three times with $200 \mu \mathrm{L}$ of PBS with 0.5\% tween-20 (PBST). We added $100 \mu \mathrm{L}$ of primary antibody, diluted 1:200, to each well and incubated the plates for 2 hours at room temperature. After washing, we added $100 \mu \mathrm{L}$ of appropriate HRPconjugated secondary antibodies, diluted 1:5000, to each well and incubated the plate for 2 hours at room temperature. The wells were washed three times with $200 \mu \mathrm{L}$ of PBST, and $100 \mu \mathrm{L}$ of o-phenylenediamine dihydrochloride (OPD) substrate solution (i.e., $26 \mathrm{mg}$ OPD, $9 \mathrm{~mL}$ deionized water, $1 \mathrm{~mL} \mathrm{H}_{2} \mathrm{O}_{2}$ ) was added to each well. Sample wells were incubated with the OPD substrate solution for $30 \mathrm{~min}$ in the dark. The color reaction was stopped with $2.5 \mathrm{M} \mathrm{H}_{2} \mathrm{SO}_{4}$. The absorbance of each well was measured at $490 \mathrm{~nm}$ with a Cary 60 UV/Vis spectrophotometer.

\section{Preparation of dendritic cells}

DCs were depleted using negative magnetic bead separation (ThermoFisher) according to manufacturer's instructions directly after removal from liquid cryopreservation. Depleted DCs were placed at $37{ }^{\circ} \mathrm{C}$ with $5 \% \mathrm{CO}_{2}$ for overnight recovery. After overnight recovery, DCs were stimulated with either LPS from Salmonella enterica serotype Minnesota or recombinant $\mathrm{HCV}$ core protein at a final concentration of $10 \mu \mathrm{g} / \mathrm{mL}$ for three, six, or twelve hours of incubation. After stimulation, DCs were harvested by centrifugation at $1000 \times \mathrm{g}$ for $5 \mathrm{~min}$ at $4{ }^{\circ} \mathrm{C}$. DCs were washed once with ice-cold PBS and centrifuged at $1000 \mathrm{x} \mathrm{g}$ for $8 \mathrm{~min}$ at $4{ }^{\circ} \mathrm{C}$. The PBS was discarded, and DCs were re-suspended in $500 \mu \mathrm{L}$ ice-cold IP lysis buffer (ThermoFisher). The cell lysates were incubated on ice for 5 min with gentle agitation. Thereafter, cell lysates were centrifuged at 13,000 x $g$ for $10 \mathrm{~min}$ and lysates were stored at $-80{ }^{\circ} \mathrm{C}$ until analysis.

\section{Determination of $\mathrm{Bcl}-2$ levels in depleted dendritic cells exposed to HCV core protein using a fluorescent immunoassay}

DC lysates were quantified using the Bradford assay. We diluted whole cell lysates to $20 \mu \mathrm{g} / \mathrm{mL}$. Fifty microliters of diluted sample were used to coat the wells of a 96-well microplate for 2 hours at room temperature. We removed the samples from the wells and blocked the coated wells with blocking buffer (1\% BSA in PBST) for 1 hour at room temperature. Sample wells were washed three times with $200 \mu \mathrm{L}$ of PBST. We added both APC-labeled BDCA2 (Miltenyi Biotec) and mouse monoclonal anti-Bcl-2 (Santa Cruz Biotechnology, Dallas, TX), both diluted 1:250 to each well for 2 hours at room temperature. Sample wells were washed three times with $200 \mu \mathrm{L}$ of PBST. We added Alexa Fluor 488-labeled goat anti-mouse secondary antibody (1:5000) to each well for 2 hours at room temperature. Sample wells were washed three times with $200 \mu \mathrm{L}$ of PBST. Fluorescent intensity was measured using a Cytation 5 Cell Imaging Multi-Mode Reader.

\section{Statistical Analysis}

We examined the relative differences in the average protein expression level using SigmaPlot, version 12.5. Protein expression measured after overnight recovery from cryopreservation serve as baseline protein expression levels. We also determined whether protein expression varied according to incubation time 
or the type of antigenic stimulation using an analysis of variance (ANOVA) test. To determine which groups differed, we applied the Holm-Sidak method for multiple pairwise comparisons. Pairwise comparisons were made against protein levels of unstimulated PBMCs or unstimulated DCs as control protein levels. The relative percent change was also determined between each incubation period for caspase- 3 and Bcl-2 expression. Error bars display the standard deviation. A $p$ value $<0.05$ was considered statistically significant.

\section{Results and Discussion \\ Plasmacytoid dendritic cell frequency}

In chronic HCV infection, several have observed that $\mathrm{pDC}$ frequency and function has been diminished. One research group has suggested that the decrease in peripheral blood pDC numbers is due to increasing migration of pDCs to the liver, where HCV infection predominates [4]. Although this is a plausible explanation for the decrease in $\mathrm{pDC}$ frequency, this still does not explain why pDC function is altered during chronic HCV infection. Therefore, we investigated if the increase in TRAIL, TRAIL-R1, and TRAIL-R2 that we observed in the total PBMC population (data not shown) correlated with pDC death receptor expression.

To begin our analysis, we determined if recombinant HCV core protein altered the number of pDCs. We used pDC markers, BDCA2 and BDCA4, to immunophenotype pDCs. pDCs are the only cells within the PBMC population to express BDCA2 and BDCA4. As Fig. 3(a) displays, there was a $\sim 0.02 \%$ change between baseline pDC numbers and pDCs after three hours of incubation. Furthermore, after six hours of incubation, unstimulated PBMCs displayed similar ( $\sim 0.02 \%$ difference) numbers of pDCs compared to baseline and unstimulated PBMCs after three hours of incubation. This difference was also similar to the differences ( $\sim 0.03 \%$ cells) between pDCs measured after three hours of incubation compared to six hours of incubation. Moreover, although very small, PBMCs stimulated with either LPS or recombinant HCV core protein showed a $\sim 0.01 \%$ increase in the number of pDCs after six hours of incubation compared to baseline pDCs. Similarly, there was only a slight difference $(\sim 0.03 \%)$ between pDCs measured after twelve hours of incubation compared to baseline pDCs (Fig. 3(a)). To add, pDCs measured after twelve hours of incubation only showed a $\sim 0.05 \%$ increase in the number of pDCs compared to those measured after six hours. Interestingly, the number of pDCs for both unstimulated and stimulated PBMCs was increased by approximately $0.06 \%$ after twelve hours of incubation compared to pDCs after three hours of incubation. Nevertheless, pDCs stimulated with either LPS or recombinant HCV core protein displayed a reduction in the number of pDCs ( 0.02\%) compared to unstimulated pDCs after twelve hours of incubation.

The difference in the number of pDCs in unstimulated PBMCs and stimulated PBMCs was not confirmed by two-way ANOVA (Fig. 3(b)). We examined if there were any differences in the number of pDCs according to incubation time or the type of antigenic stimulus. Two-way ANOVA showed that the statistically significant differences in the number of pDCs was associated with incubation time $(p=0.016)$. We performed post-hoc multiple pairwise comparisons

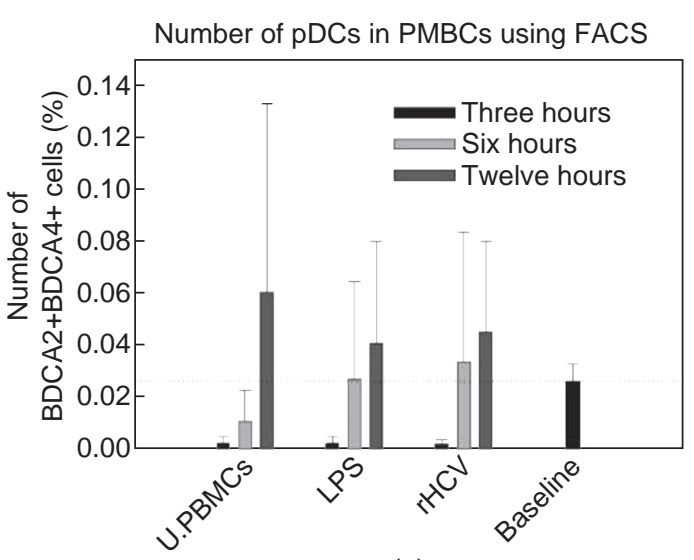

(a)

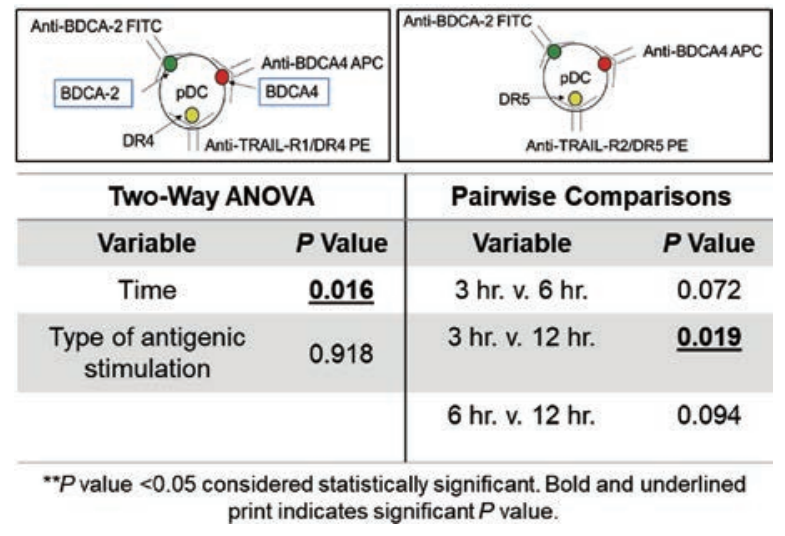

(b)

Fig. 3 (a) Number of plasmacytoid dendritic cells (pDCs). Bar graphs represent the average number of pDCs from three replicates in 3, 6, and 12 hours. Error bars show the standard deviation (SD). (b) Two-way analysis of variance (ANOVA) for number of pDCs, followed by multiple pairwise comparisons using the Holm-Sidak method for the different incubation periods (i.e., 3, 6, or 12 hours). 
and observed that pDCs measured after three hours of incubation differed significantly from $\mathrm{pDCs}$ measured after twelve hours of incubation $(p=0.019)$. There were no significant differences in pDCs stimulated with either LPS or recombinant HCV core protein between unstimulated pDCs $(p=0.918)$. Therefore, recombinant $\mathrm{HCV}$ core protein stimulation does not have a direct effect on the number of pDCs in the PBMC population.

\section{TRAIL-R1 and TRAIL-R2 expression in plasmacytoid dendritic cells using multicolor FACS}

We investigated whether recombinant HCV core protein caused increased TRAIL-R1 or TRAIL-R2 expression on pDCs, which may increase their susceptibility to apoptosis via the TRAIL pathway. We incubated PBMCs with recombinant HCV core protein for various time periods (i.e., three, six, or twelve hours). PBMCs incubated with LPS and unstimulated PBMCs served as control samples. We also determined the level of TRAIL-R1 and TRAIL-R2 expression directly after an overnight recovery period from cryopreservation to serve as baseline. Fig. 4 displays representative density plots for pDCs expressing TRAIL-R1 and TRAIL-R2 after three hours of incubation.

We examined the number of pDCs (identified as either BDCA2+ or BDCA4+) expressing TRAIL-R1 or TRAIL-R2 expression. Overall, we observed that PBMCs stimulated with either LPS or recombinant HCV core protein caused an increase ( $\sim 0.02 \%$ cells) in TRAIL-R1 expression on pDCS after three hours of incubation in BDCA2+ cells in comparison to unstimulated PBMCs (Fig. 5(a)). We observed this increase only in BDCA2+ cells. TRAIL-R1 expression was similar ( 0.002\%) for all BDCA4+ cells after three hours of incubation, except the number of BDCA4+TRAIL-R1+ cells in PBMCs stimulated with LPS was lower ( average $0.001 \%$ cells) than the number of BDCA4+TRAIL-R1+ cells in unstimulated PBMCs and PBMCs stimulated with recombinant HCV core protein (Fig. 5(a)). BDCA4+ cells at baseline did not have any TRAIL-R1 expression. Moreover, TRAIL-R2 expression was not comparable to the trend in TRAIL-R1 expression after three hours of incubation. TRAIL-R2 expression in BDCA2+ cells was lower ( $\sim 0.03 \%$ cells) than TRAIL-R1 expression in BDCA2+ cells after three hours of incubation. For BDCA4+ cells, TRAIL-R2 expression was approximately the same $(\sim 0 \%)$ on all pDCs, regardless of stimulation after three hours of incubation. Nevertheless, PBMCs stimulated with recombinant HCV core protein displayed the highest $(\sim 0.01 \%$ difference) number of pDCs with either TRAIL-R1 or TRAIL-R2 expression after three hours of incubation compared to unstimulated PBMCs.

Interestingly, TRAIL-R1 expression decreased $\sim 0.02 \%$ cells in both BDCA2+ and BDCA4+ cells in PBMCs stimulated with recombinant HCV core protein after six hours of incubation. We observed a decrease ( $\sim 0.03 \%$ cells) in TRAIL-R1 expression in BDCA2+ cells in PBMCs stimulated with LPS after six hours. However, for BDCA4+ cells, TRAIL-R1 expression remained constant ( $\sim 0.001 \%$ cells) between three hours and six hours of incubation. Contrarily, unstimulated PBMCs exhibited a $\sim 0.01 \%$ cells increase in the number of BDCA2+TRAIL-R1+ cells after six hours of incubation. Similarly, TRAIL-R1 expression in BDCA4+ cells remained constant ( $0.001 \%$ cells) between three hours and six hours of incubation for unstimulated PBMCs. TRAIL-R2 expression also showed a similar pattern compared to TRAIL-R1 expression after six hours of incubation. There was a $\sim 0.01 \%$ cells increase in TRAIL-R2 expression in both BDCA2+ and BDCA4+ cells in both unstimulated PBMCs and PBMCs stimulated with LPS. However, TRAIL-R2 expression in BDCA2+ and BDCA4+ cells decreased $(\sim 0.002 \%$ cells) in PBMCs stimulated with recombinant HCV core protein (Fig. 5(a)).

The trend in death receptor expression was different for cells incubated for twelve hours. In BDCA2+ cells, TRAIL-R1 expression remained constant $(\sim 0.006 \%$ cells) for both unstimulated PBMCs and stimulated PBMCs. Nonetheless, unstimulated PBMCs displayed a larger number $(\sim 0.01 \%$ cells $)$ of BDCA2+ cells with TRAIL-R1 expression. Furthermore, TRAIL-R1 expression in BDCA4+ cells in PBMCs stimulated with LPS or recombinant HCV core protein was higher ( $0.003 \%$ cells) compared to unstimulated PBMCs. The number of BDCA4+TRAIL-R1+ cells was also increased $\sim 0.002 \%$ after twelve hours of incubation relative to six hours in both PBMCs stimulated with LPS and recombinant HCV core protein. TRAIL-R2 expression in BDCA2+ cells for both unstimulated PBMCs and PBMCs stimulated with recombinant HCV core protein were similar ( $\sim 0.010 \%$ and $\sim 0.003 \%$, respectively) between six hours and twelve hours of incubation. In contrast, the number of BDCA2+TRAIL-R2+ cells increased $(\sim 0.01 \%$ cells $)$ in PBMCs stimulated with LPS after 
FACS: TRAIL-R1 or TRAIL-R2 expression in pDCs

TRAIL-R1

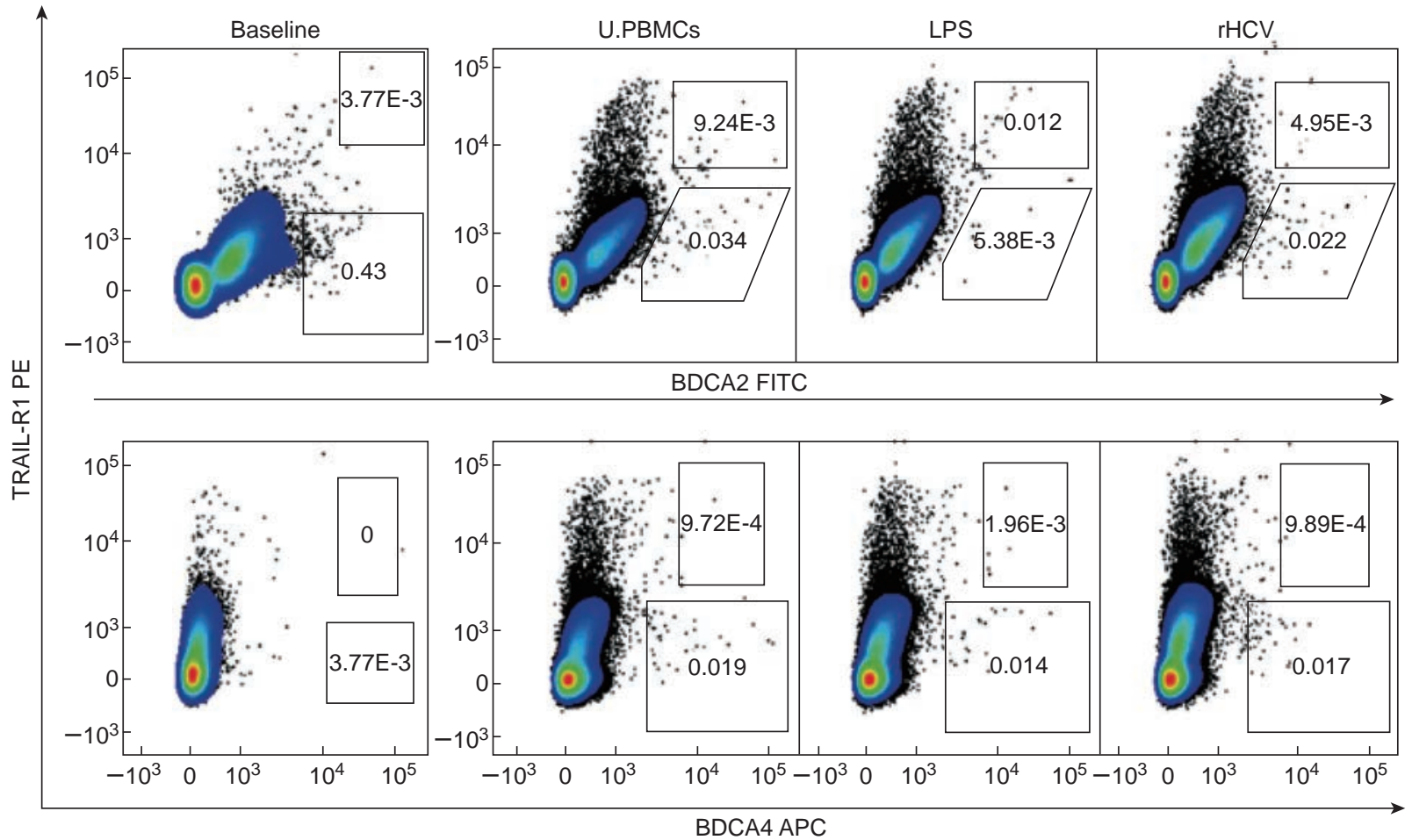

TRAIL-R2

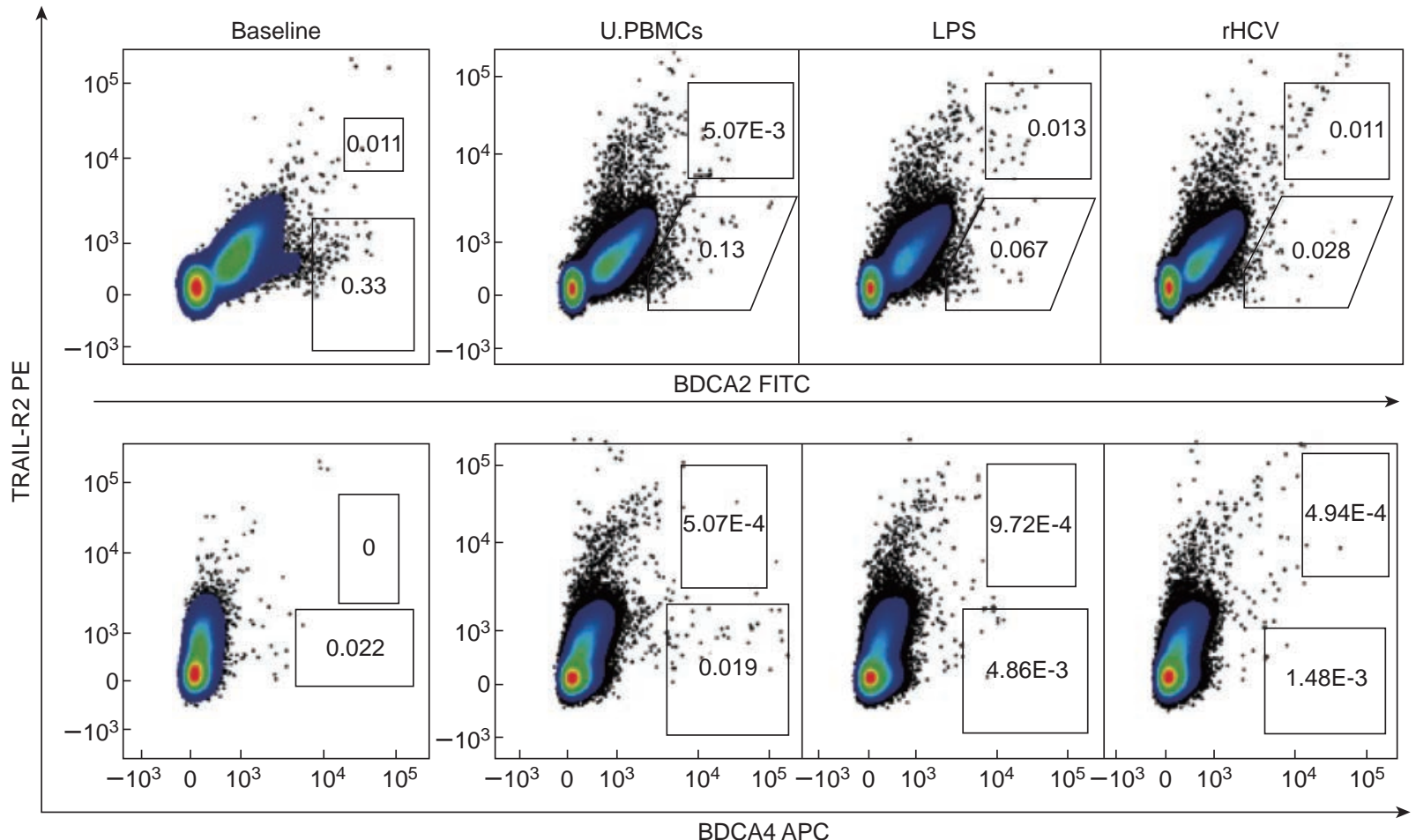

Fig. 4 Density plots of multicolor FACS analysis of (a) TRAIL-R1 and (b) TRAIL-R2 in unstimulated and stimulated pDCs after three hours of incubation. Baseline was the time control $(t=0)$. Cells were examined for BDCA2, BDCA4, TRAIL-R1 and TRAIL-R2 expression. The numbers in each box represent the percentage of positive cells. Abbreviations: U.PBMCs, unstimulated PBMCs; LPS, lipopolysaccharide; rHCV, recombinant HCV core protein.

twelve hours of incubation. Moreover, BDCA4+ cells presented a different trend after twelve hours of incubation. The number of BDCA4+TRAIL-R2+ cells decreased ( $0.003 \%$ cells) in unstimulated PBMCs. However, the number of BDCA4+TRAIL-R2+ cells in PBMCs stimulated with recombinant HCV core 


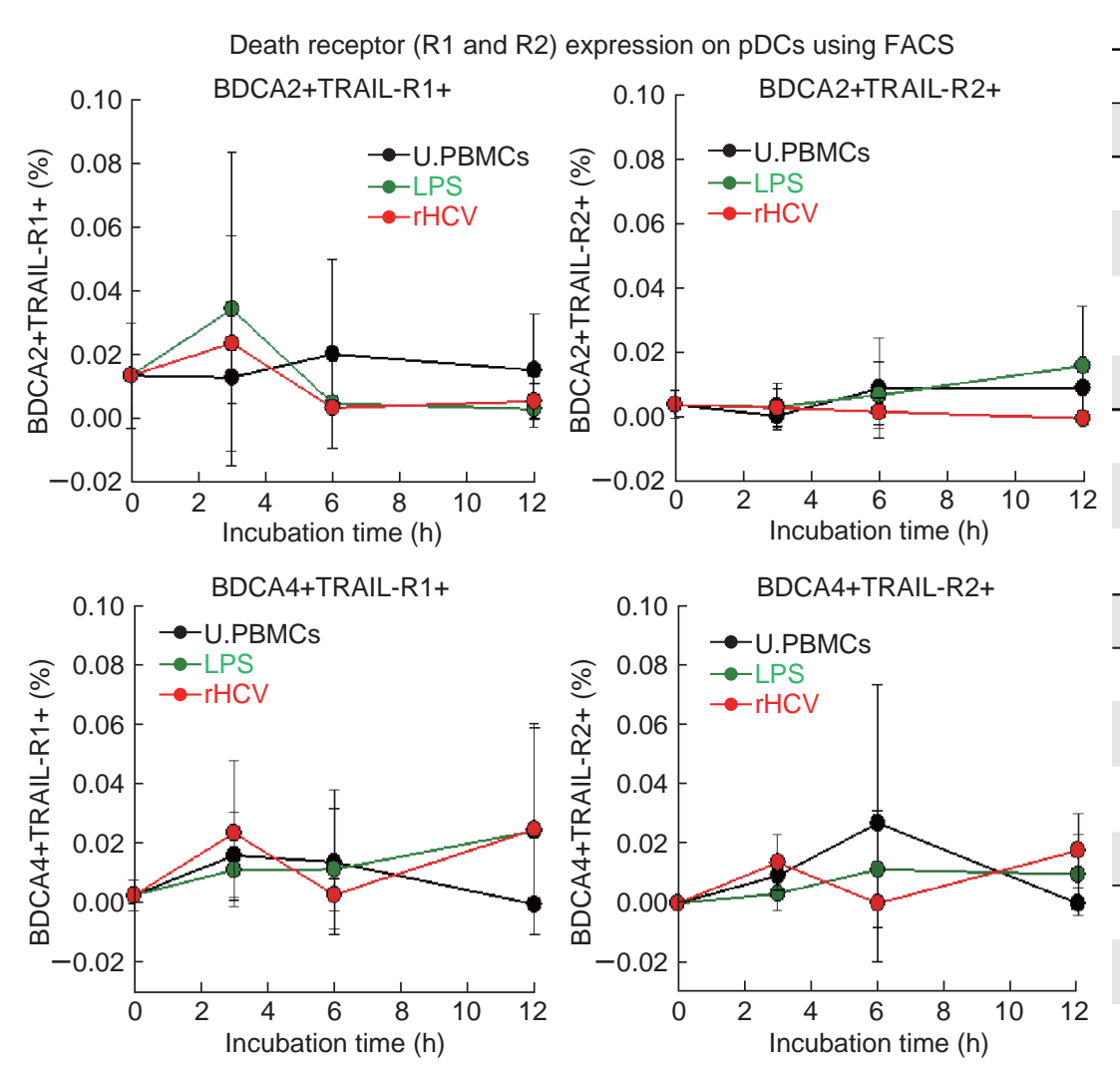

(a)

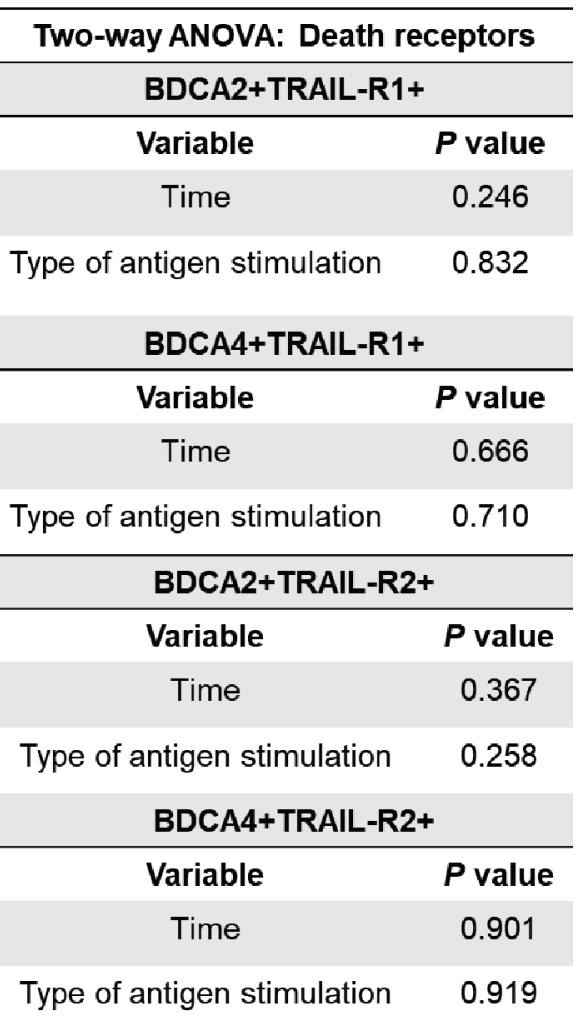

(b)

Fig. 5 Time trend analysis of death receptor expression in pDCs. Each point represents the average number of cells expressing TRAIL-R1, TRAIL-R2, or TRAIL determined by FACS. Time 0 is the level of either TRAIL-R1, TRAIL-R2, or TRAIL expression after overnight recovery from cryopreservation. Time 0 represents the time control. Unstimulated PBMCs represent control samples.

protein increased $(\sim 0.002 \%$ cells) after twelve hours of incubation. There was no change in the number of BDCA4+TRAIL-R2+ cells in PBMCs stimulated with LPS after twelve hours of incubation. A heatmap representation of death receptor expression in pDCs is shown in Fig. 6.

Because of the differences observed in TRAIL-R1 and TRAIL-R2 expression on BDCA2+ and BDCA4+ cells, we determined if the level of protein expression varied according to incubation time or the type of antigenic stimulation. We performed a two-way ANOVA to investigate these differences. We did not observe any statistically significant differences in either TRAIL-R1 or TRAIL-R2 expression on either BDCA2+ or BDCA4+ cells (Fig. 5(b)).

\section{TRAIL-R1 and TRAIL-R2 expression in plasmacytoid dendritic cells using image cytometry}

A benefit of using an image cytometer is that a fluorescent image can be obtained for each cell expressing the marker of interest. We examined the expression of TRAIL-R1 and TRAIL-R2 in pDCs (identified as BDCA2+ or BDCA4+ expression) after various incubation periods (i.e., three, six, or twelve hours). Interestingly, BDCA4+ cells did not display any death receptor expression in any of the PBMCs (Fig. 7). This observation was similar to multicolor FACS (Fig. 6 and 7). Death receptors were co-localized on BDCA2+ cells. TRAIL-R1 was higher in PBMCs stimulated with recombinant HCV core protein compared to unstimulated PBMCs after three hours of incubation. TRAIL-R2 expression was similar in both unstimulated PBMCs and PBMCs stimulated with recombinant HCV core protein after three hours of incubation. Furthermore, the fluorescent images showed that there was a decrease in both TRAIL-R1 and TRAIL-R2 expression in BDCA2+ cells in PBMCs stimulated with recombinant HCV core protein after six hours of incubation, relative to unstimulated PBMCs. However, the fluorescent images displayed that there was an increase in TRAIL-R1 and TRAIL-R2 expression after twelve hours of incubation for PBMCs stimulated with recombinant HCV core protein compared to unstimulated PBMCs. 


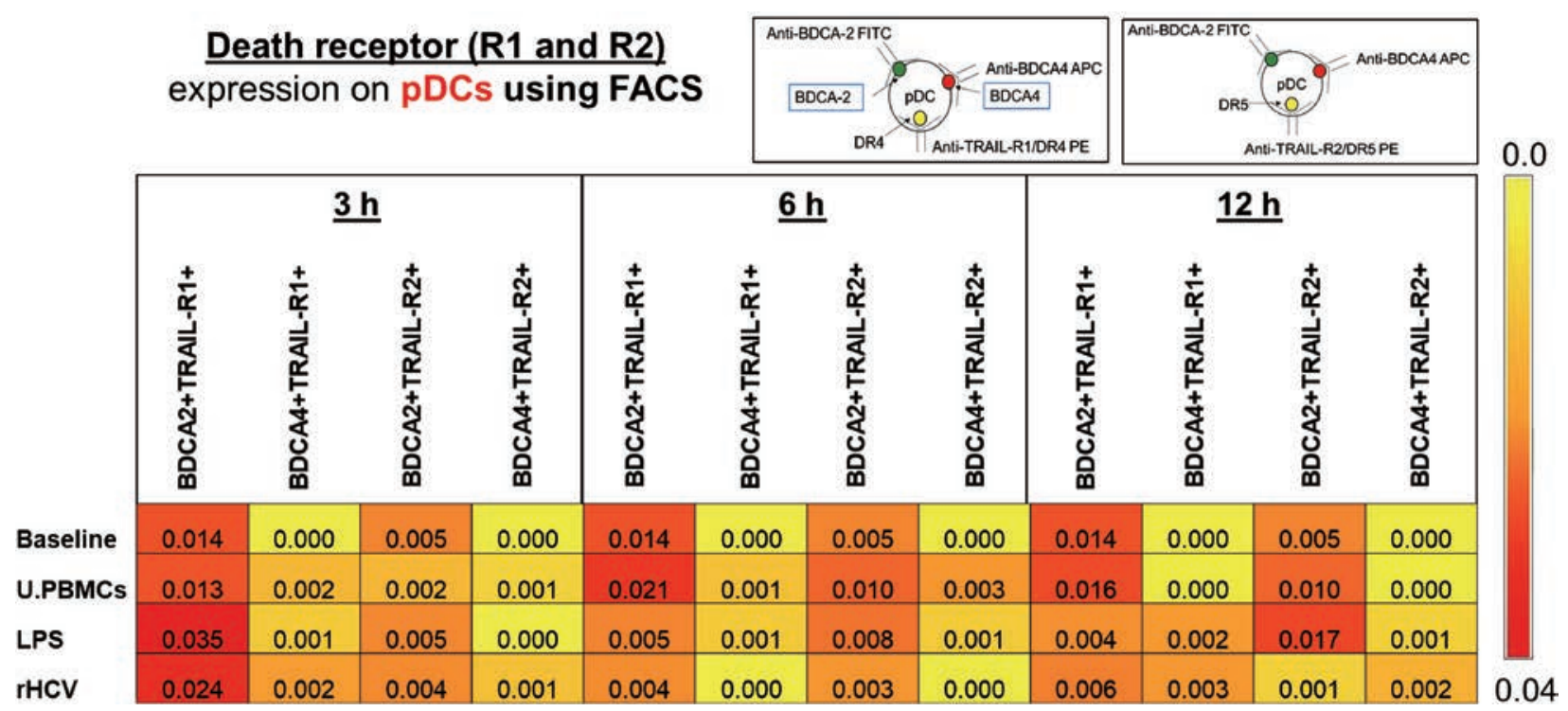

Fig. 6 Heat map representation displaying average FACS percentages of TRAIL-R1 and TRAIL-R2 expression in pDCs. Unstimulated PBMCs were used as control PBMCs. Baseline is the time control $(t=0)$. Each color matrix represents the average percentage of cells expressing either TRAIL-R1 or TRAIL-R2 from three replicates in 3, 6, and 12-hour PBMCs.

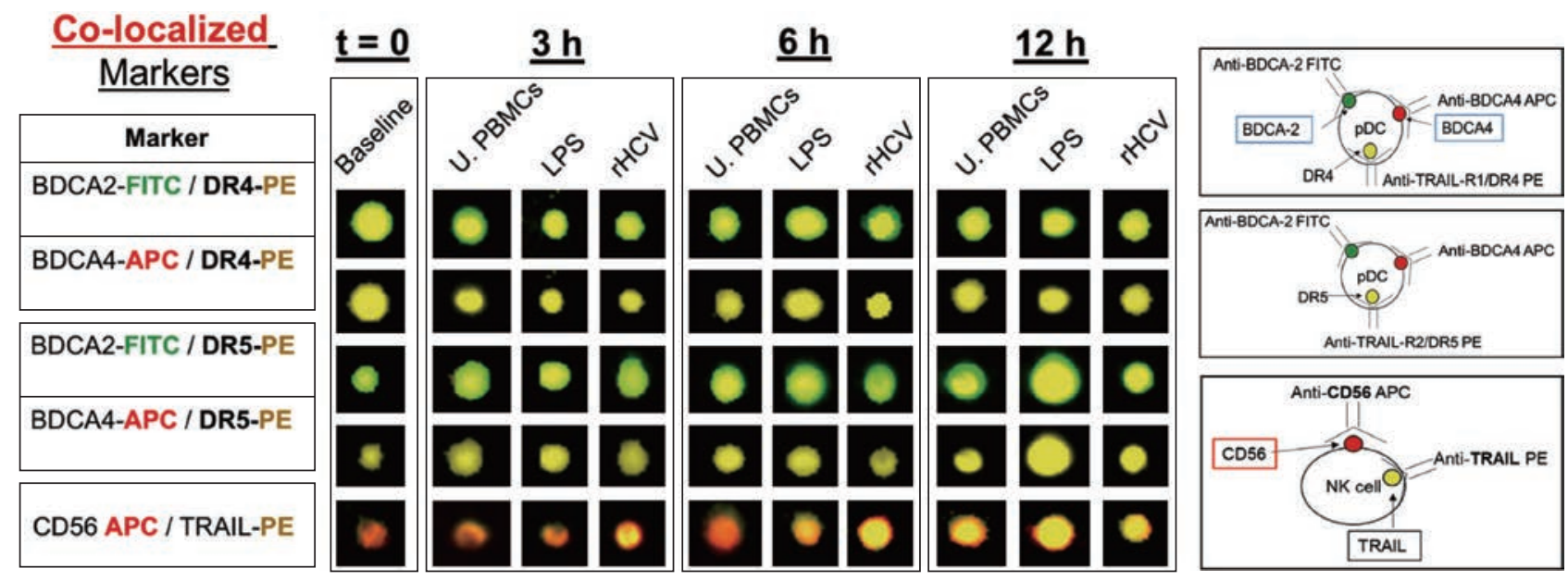

Fig. 7 Fluorescent images of co-localization of death receptors or TRAIL in pDCs and NK cells using image cytometry. Unstimulated PBMCs were used as control samples. Baseline was the time control $(t=0)$. Cells were examined for BDCA2, BDCA4, TRAIL-R1/ DR4, TRAIL-R2/DR5, and TRAIL expression. Abbreviations: U. PBMCs, unstimulated PBMCs; LPS, lipopolysaccharide; rHCV, recombinant HCV core protein.

\section{Determination of relative expression of TRAIL in NK cells using multicolor FACS}

Because of the bi-directional relationship of NK cells and pDCs and the mechanism of NK cytolysis, we determined if there was an increase in TRAIL expression on NK cells (identified as CD56+ cells). We hypothesized that increased pDC apoptosis was due to increased NK cell deletion of pDCs via the TRAILmediated pathway. We examined the number of CD56+ cells in unstimulated and stimulated PBMCs. Interestingly, after three hours, all cells displayed a lower $(\sim 0.34 \%)$ number of NK cells compared to baseline (Fig. 8(a) and (b)). To add, after three hours of incubation, there was $0.07 \%$ less TRAIL expression in NK cells relative to baseline. Nonetheless, CD56+TRAIL+ expression was similar $(\sim 0.01 \%)$ in unstimulated and stimulated PBMCs.

After six hours of incubation, there was a $0.66 \%$ increase in the number of CD56+ cells in PBMCs stimulated with recombinant HCV core protein compared to unstimulated PBMCs. This increase also correlated in the number of CD56+TRAIL+ cells in PBMCs stimulated with recombinant HCV core protein. Unstimulated PBMCs and PBMCs stimulated with LPS also displayed $\sim 0.35 \%$ increase after six hours of incubation, just not as high as PBMCs stimulated with recombinant HCV core protein. Moreover, the number of CD56+ cells after 
(a)

TRAIL/Apo2L and CD56+ PBMCs

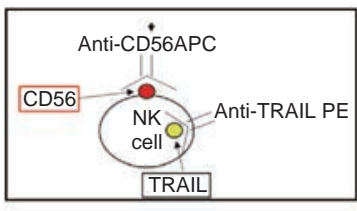

\begin{tabular}{|c|c|c|c|c|c|}
\hline & TRAIL] & Baseline & U.PBMCs & LPS & rHCV \\
\hline \multirow{2}{*}{$3 \mathrm{~h}$} & \multirow{2}{*}{$\begin{array}{l}\text { CD56+ } \\
\text { CD56+ TRAIL }\end{array}$} & 0.55 & 0.27 & 0.22 & 0.21 \\
\hline & & 0.08 & 0.01 & 0.01 & 0.01 \\
\hline \multirow{2}{*}{$6 \mathrm{~h}$} & \multirow{2}{*}{$\begin{array}{l}\text { CD56+ } \\
\text { CD56+ TRAIL }\end{array}$} & 0.55 & 0.34 & 0.36 & 0.87 \\
\hline & & 0.08 & 0.34 & 0.36 & 0.87 \\
\hline \multirow[t]{2}{*}{$12 \mathrm{~h}$} & \multirow[t]{2}{*}{ CD56+ } & 0.55 & 0.34 & 0.36 & 0.87 \\
\hline & & 0.08 & 0.20 & 0.19 & 0.22 \\
\hline
\end{tabular}
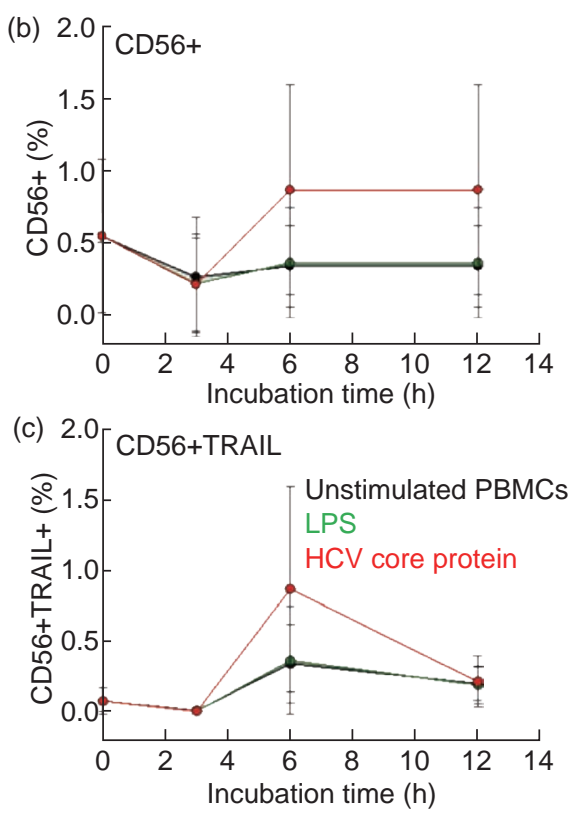

Fig. 8 (a) Heat map representation displaying average FACS percentages of CD56 and TRAIL expression. Unstimulated PBMCs were used as control PBMCs. Baseline is the time control $(t=0)$. Each color matrix represents the average percentage of cells expressing either CD56 or TRAIL from three replicates in 3, 6, and 12-hour PBMCs. Expression of CD56 and TRAIL by FACS analysis. Time trend of (b) CD56+ and (c) CD56+TRAIL+ expression. Each point represents the average percentage of cells expressing CD56 and TRAIL determined by FACS. Time 0 is the level of either CD56 or TRAIL expression after overnight recovery from cryopreservation. Time 0 represents the time control. Unstimulated PBMCs represent control samples.

twelve hours of incubation remained consistent with that observed after six hours of incubation for both unstimulated PBMCs and stimulated PBMCs. However, the percentage of CD56+TRAIL+ cells decreased $(\sim 0.17 \%)$ for both unstimulated PBMCs and PBMCs stimulated with LPS. However, PBMCs stimulated with recombinant HCV core protein experienced the most significant level of decrease ( 0.65\%) in CD56+TRAIL+ expression (Fig. 8 (a) and (c)).

\section{Determination of relative expression of TRAIL in NK cells using image cytometry}

Notably, there was a clear difference in the level of CD56+ and CD56+TRAIL+ expression in unstimulated PBMCs and stimulated PBMCs using image cytometry. Fig. 7 displays the fluorescent images for the co-localization of TRAIL on NK cells. We observed that PBMCs stimulated with recombinant $\mathrm{HCV}$ core protein exhibited higher fluorescent intensity for CD56+TRAIL+ expression than unstimulated PBMCs after three hours and six hours of incubation (Fig. 8(a) and (c)). Using multicolor FACS, we observed increases in the number of CD56+TRAIL+ cells in PBMCs stimulated with recombinant HCV core protein after six and twelve hours of incubation. Image cytometry showed similar TRAIL expression in
CD56+ cells after twelve hours of incubation for both unstimulated PBMCs and PBMCs stimulated with recombinant HCV core protein (Fig. 7).

\section{Determination of relative expression of caspase-3 and Bcl-2 in total PBMC and dendritic cells}

Because of increased death receptor expression in pDCs and increased TRAIL in NK cells, we determined if HCV core activates the Bcl-2 pathway. Notably, we did not observe a consistent trend in caspase-3 activity in either of the PBMC samples using ELISA (Fig. 9(a) and (d)). Caspase-3 activity did not significantly differ based on the time or the type of antigenic stress (Table 1). Moreover, in total PBMCs, Bcl-2 expression was similar for all PBMC samples (Fig. 9(b)). However, using ELISA, PBMCs stimulated with recombinant HCV core protein displayed the highest ( $\sim \%$ ) level of Bcl-2 expression after three hours of incubation relative to unstimulated PBMCs (Fig. 9(e)). Both PBMCs stimulated with LPS and recombinant HCV core protein displayed higher levels ( $\sim \%$ ) of Bcl-2 expression in all incubation periods relative to unstimulated PBMCs, except after six hours of incubation. To add, there was a 36\% in Bcl2 expression in all PBMC samples after six hours of incubation (Fig. 9(b)). However, after twelve hours 
ELISA: total PBMCS

Caspase-3
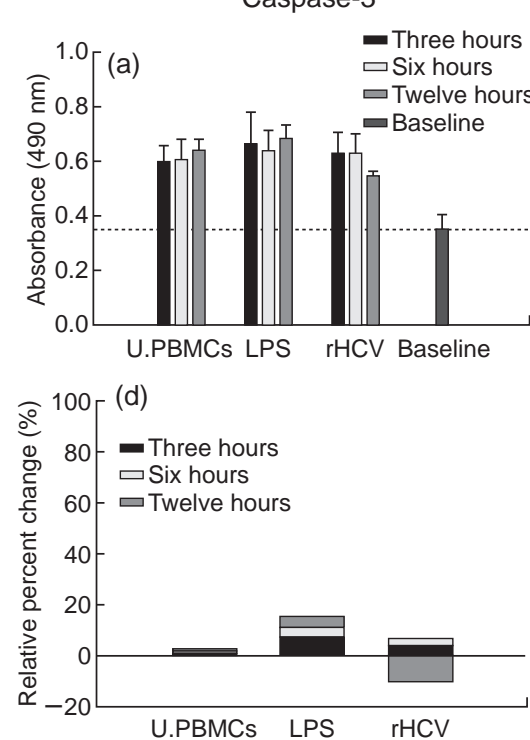

$\mathrm{Bcl}-2$
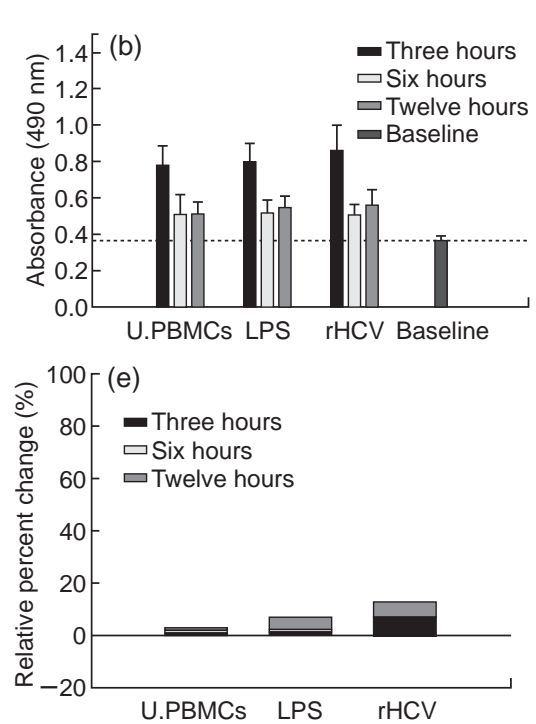

Fluorescence: dendritic cells

$\mathrm{BCl}-2$
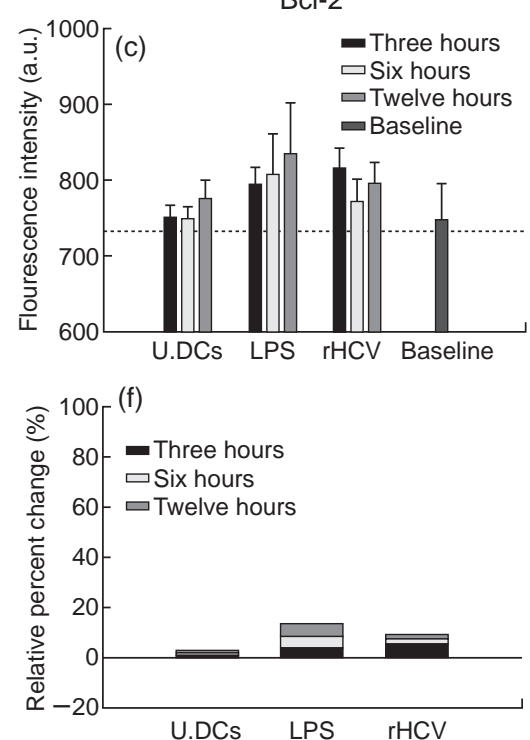

Fig. 9 Caspase-3 and Bcl-2 expression in total PBMCs and depleted dendritic cells. Semi-quantitative analysis using an indirect ELISA method to determine (a) caspase-3 or (b) Bcl-2 in total PBMC whole cell lysates. Semi-quantitative analysis using a fluorescent immunoassay method to determine (c) Bcl-2 in depleted dendritic cell whole cell lysates. Bar graphs represent the average absorbance of OPD product formation at $490 \mathrm{~nm}$ or mean fluorescent intensity. Error bars show the standard deviation. Stacked proportion graphs indicating the relative percent change in (d) caspase-3 and (e) Bcl-2 in total PBMC whole cell lysates and (f) Bcl-2 in depleted dendritic cells. U. PBMCs are set as the reference group $=1$.

of incubation, there was a $\sim 5 \%$ increase in Bcl-2 expression in PBMCs (Fig. 10).

Two-way ANOVA showed that Bcl-2 expression differed significantly between the different incubation periods $(p \leq 0.001)$. However, we did not find any significant difference in Bcl-2 expression between unstimulated and stimulated PBMCs $(p=0.22)$. Using the Holm-Sidak method, there were significant differences between three hours and six hours of incubation as well as between three hours and twelve hours of incubation (Table 1).

On the other hand, the trend in Bcl-2 expression in depleted DCs was not the same as the one observed in total PBMCs. For example, there was a slight increase ( $1 \%)$ in Bcl-2 expression with time in all DCs, except DCs stimulated with recombinant HCV core protein using fluorescence (Fig. 10). After three hours of incubation both DCs stimulated with LPS or recombinant HCV core protein displayed higher ( $\sim \%$ and 5\%, respectively) Bcl-2 expression than unstimulated DCs (Fig. 9(c) and (f)). DCs stimulated with recombinant HCV core protein decreased $\sim 4 \%$ between three hours and six hours of incubation (Fig. 10). Nonetheless, DCs stimulated with LPS and recombinant $\mathrm{HCV}$ core protein remained higher $(\sim 5 \%$ and $2 \%$, respectively) than unstimulated DCs after six hours of incubation (Fig. 10). Furthermore, DCs stimulated with either LPS or recombinant HCV core protein exhibited higher ( $\sim 5 \%$ and $2 \%$, respectively) Bcl-2 levels than unstimulated DCs after twelve hours of incubation (Fig. 9(c) and (f)). To add, there was $~ 2 \%$ increase in Bcl-2 expression in all DCs after twelve hours of incubation (Fig. 10).

Two-way ANOVA showed that Bcl-2 expression did not differ significantly according to time. Bcl-2 expression was also only marginally significant for the type of antigenic stimulation $(p=0.046)$. However, after performing multiple pairwise comparisons, there was no significant difference in Bcl-2 expression between the different samples (Table 1).

A major issue regarding the defect in pDCs is whether HCV infection directly inhibits pDC function because HCV is not known to establish infection in pDCs. We investigated if recombinant HCV core protein caused increased TRAIL-R1 or TRAIL-R2 expression on pDCs and increased TRAIL in NK cells. Overall, we did not observe a significant difference in the number of pDCs between unstimulated PBMCs and PBMCs stimulated with recombinant HCV core protein. A possible explanation for this observation could be that HCV core protein does not have a direct effect on pDC frequency. Other HCV-related 


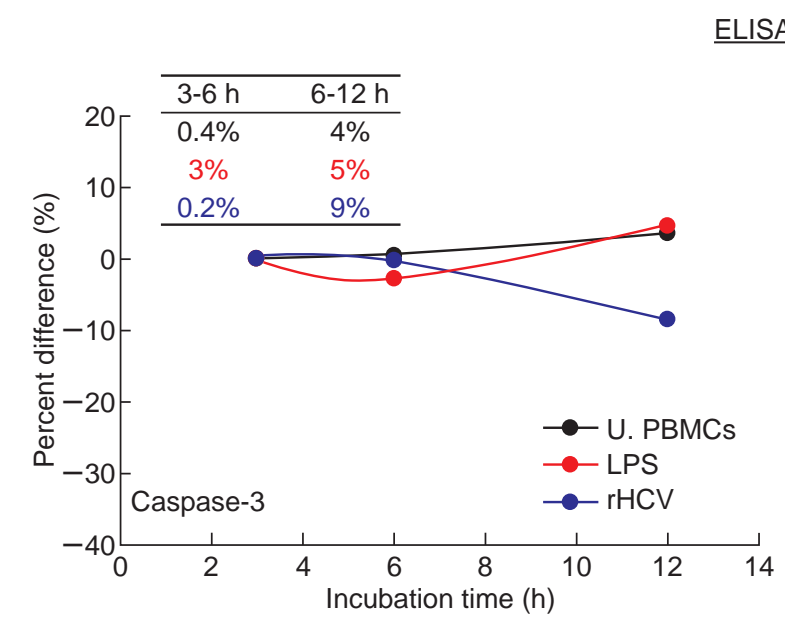

LISA: Total PBMCs

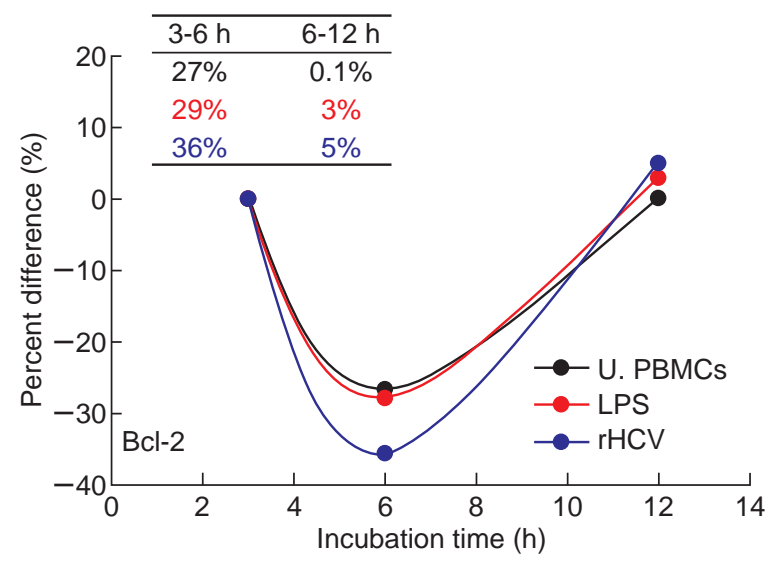

Fluorescence: dendritic cells

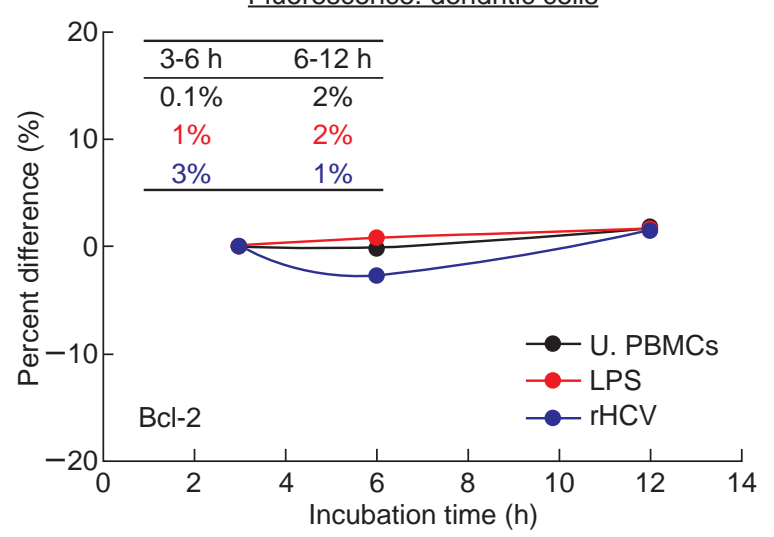

Fig. 10 Percent difference in the expression of caspase-3 and Bcl-2 in total PBMCs and depleted dendritic cells between three, six, and twelve hours of incubation in unstimulated and stimulated PBMCs. Abbreviation: ELISA, enzyme-linked immunosorbent assay; U. PBMCs, unstimulated PBMCs; LPS, lipopolysaccharide; rHCV, recombinant HCV core protein.

Table 1 Two-way ANOVA: Bcl-2 and caspase-3 expression. Two-way ANOVA for caspase-3 and Bcl-2 expression in total PBMCs and depleted DCs, followed by multiple pairwise comparisons using the Holm-Sidak method. Abbreviation: PBMCs, peripheral blood mononuclear cells; U.PBMCs, unstimulated PBMCs; LPS, lipopolysaccharide; rHCV, recombinant HCV core protein. $P$ value $<0.05$ was considered statistically significant. Bold and underlined print indicates a significant $P$ value.

\begin{tabular}{|c|c|c|c|c|c|c|c|c|c|}
\hline \multirow{2}{*}{\multicolumn{2}{|c|}{$\begin{array}{c}\text { Total peripheral blood } \\
\text { mononuclear cells }\end{array}$}} & \multicolumn{4}{|c|}{ Total peripheral blood mononuclear cells } & \multicolumn{4}{|c|}{ Depleted dendritic cells } \\
\hline & & \multicolumn{2}{|c|}{ Bcl-2: Two-way ANOVA } & \multicolumn{2}{|c|}{ Pairwise comparisons } & \multicolumn{2}{|c|}{ Bcl-2: Two-way ANOVA } & \multicolumn{2}{|c|}{ Pairwise comparisons } \\
\hline Variable & $P$ value & Variable & $P$ value & Variable & $P$ value & Variable & $P$ value & Variable & $P$ value \\
\hline Time & 0.957 & Time & $<\underline{0.001}$ & 3 hr. v. 6 hr. & $<\underline{0.001}$ & Time & 0.313 & U.DCs v. LPS & $\underline{0.039}$ \\
\hline \multirow[t]{2}{*}{$\begin{array}{l}\text { Type of antigenic } \\
\text { stimulation }\end{array}$} & 0.281 & $\begin{array}{l}\text { Type of antigenic } \\
\text { stimulation }\end{array}$ & 0.220 & 3 hr. v. 12 hr. & $<\underline{0.001}$ & $\begin{array}{l}\text { Type of antigenic } \\
\text { stimulation }\end{array}$ & $\underline{0.046}$ & U.DCs v. rHCV & 0.067 \\
\hline & & & & 6 hr. v. 12 hr. & 0.255 & & & & \\
\hline
\end{tabular}

proteins may have a direct effect on the number of pDCs observed in those with HCV infection. To add, several investigators have found that $\mathrm{pDC}$ frequency is reduced in those with chronic HCV infection [1-3]. There is also evidence that this decrease may be due to pDC apoptosis in those with chronic HCV infection [5]. Such evidence was based upon increased expression of TNF- $\alpha$ and IL-10 in monocytes [5]. However, this observation does not explain the increased expression of TRAIL-R1 or TRAIL-R2 on pDCs. Thus, our research group is also carrying out studies on pDC frequency in $\mathrm{HCV}$ infection to investigate the impact of TRAIL-mediated apoptosis of pDCs in the absence of monocytes and determine if TNF- $\alpha$ or IL-10 plays a role in the induction of this pathway. We hypothesize that free HCV core protein causes the upregulation of TRAIL-R1 and TRAIL-R2 in immature peripheral pDCs, causing these pDCs to be susceptible to TRAIL- 
mediated death induced by NK cells. We note that the investigation of $\mathrm{pDC}$ frequency in $\mathrm{HCV}$ infection currently underway and will be reported in due course.

Both TRAIL-R1 and TRAIL-R2 were more prominent on BDCA2+ cells compared to BDCA4+ cells in all pDCs, regardless of stimulation. The approximate $0.03 \%$ cell difference observed in the level of BDCA2 or BDCA4 expression and the level of death receptor expression on either BDCA2 or BDCA4+ cells may be attributed to the function of BDCA2 and BDCA4. BDCA2 is a calcium-dependent type II lectin receptor involved in antigen capture and in the regulation of type I IFN production [18]. BDCA4, on the other hand, is important for initiating immune responses and directing pDCs to lymphatics $[19,20]$. Thus, the lack of addition of Fms-related tyrosine kinase 3 ligand to PBMC cultures may contribute to impaired pDC development, increasing co-localization of death receptors on BDCA2+ cells versus BDCA4+ cells.

Furthermore, we did not observe a similar trend in TRAIL-R2 expression in pDCs compared to TRAIL-R1 expression in pDCs. There was a difference of approximately $0.03 \%$ cells that were TRAIL-R1+ versus TRAIL-R2+ for all pDCs (i.e., baseline, unstimulated PBMCs, PBMCs stimulated with LPS, and PBMCs stimulated with recombinant HCV core protein. Because the density of TRAIL-R1 on pDCs is higher than TRAIL-R2, we conclude that induction of the TRAIL pathway in pDCs is most likely via engagement of TRAIL with TRAIL-R1.

It is also interesting to note that after six hours of incubation, both CD56+ expression and TRAIL expression on CD56+ cells increased approximately 0.53\% in PBMCs stimulated with recombinant HCV core protein relative to unstimulated PBMCs. CD56 is typically used as a phenotypic activation marker, whereby upon the activation of NK cells, there is increased CD56 expression. Notably, we observed a substantial increase in CD56 expression (average of $\sim 0.66 \%$ cells) and in TRAIL expression (average of $\sim 0.86 \%$ cells) on CD56+ cells after six hours of incubation in PBMCs stimulated with recombinant HCV core protein. Hence, HCV core protein increases cytolytic activity of NK cells within twelve hours of exposure. In conjunction with an increase in death receptor expression on pDCs within twelve hours, increased cytotoxic activity in NK cells makes pDCs susceptible to NK deletion by the TRAIL pathway. While our data show that pDCs may be vulnerable to
NK cell-mediated apoptosis via the TRAIL pathway, it is important to determine if pDC maturation plays a role in this mechanism. Our research group is also investigating the role of pDC maturation in NK cellmediated apoptosis and the results will be reported in due course.

The extrinsic and intrinsic pathways of apoptosis can be activated either concurrently or separately in target cells [21-23]. To add, Bcl-2 protein is shown to be essential for pDC survival [24]. Thus, we investigated whether increased TRAIL-R1 or TRAIL-R2 expression occurred with decreased Bcl-2 expression in pDCs stimulated with HCV core protein. Herein, we report that $\mathrm{HCV}$ core protein did not significantly alter Bcl2 expression in pDCs within 12 hours, suggesting that the intrinsic pathway of apoptosis is not activated with increased TRAIL-R1 or TRAIL-2 expression in pDCs. However, it is important to note that activation of the intrinsic pathway may involve other Bcl-2 family proteins. Therefore, our research group is investigating the relationship between increased TRAIL-R1 or TRAIL-R2 in pDCs and decreased expression of antiapoptotic proteins of the $\mathrm{Bcl}-2$ family after exposure to HCV core protein.

\section{Conclusions}

In this study, we demonstrated that HCV core protein increases the number of pDCs expressing TRAIL-R1 and TRAIL-R2, in particularly TRAIL-R1 expression within twelve hours of exposure ( $0.01 \%)$. We also show that HCV core protein increases $\sim 0.86 \%$ NK cell cytotoxic activity, which enhances pDC susceptibility to TRAIL-mediated apoptosis. Our results suggest that increased TRAIL expression on NK cells and increased death receptor expression on pDCs may alter the bi-directional relationship between NK cells and pDCs. Alteration to the bi-directional relationship between NK cells and pDCs may prevent a robust innate immune response against $\mathrm{HCV}$, contributing to viral persistence within HCV-infected individuals.

\section{Acknowledgments}

The authors would like to thank the School of Computer, Mathematical, and Natural Sciences for partially funding this research. Flow cytometry analyses were performed at the University of Maryland Marlene and Stewart Greenebaum Comprehensive 
Cancer Center Flow Cytometry Shared Service.

\section{Conflict of Interests}

The authors declare that no competing interest exists.

\section{References}

[1] A. Ulsenheimer, J.T. Gerlach, M.C. Jung, et al., Plasmacytoid dendritic cells in acute and chronic hepatitis C virus infection. Hepatology, 2005, 41(3): 643-651.

[2] N. Goutagny, C. Vieux, E. Decullier, et al., Quantification and functional analysis of plasmacytoid dendritic cells in patients with chronic hepatitis $\mathrm{C}$ virus infection. The Journal of Infectious Diseases, 2004, 189(9): 1646-1655.

[3] A.M. Wertheimer, A. Bakke, and H.R. Rosen, Direct enumeration and functional assessment of circulating dendritic cells in patients with liver disease. Hepatology, 2004, 40(2): 335-345.

[4] J. Nattermann, H. Zimmermann, A. Iwan, et al., Hepatitis $\mathrm{C}$ virus $\mathrm{E} 2$ and $\mathrm{CD} 81$ interaction may be associated with altered trafficking of dendritic cells in chronic hepatitis $\mathrm{C}$. Hepatology, 2006, 44(4): 945-954.

[5] A. Dolganiuc, S. Chang, K. Kodys, et al., Hepatitis C virus (HCV) core protein-induced, monocyte-mediated mechanisms of reduced IFN- $\alpha$ and plasmacytoid dendritic cell loss in chronic HCV infection. The Journal of Immunology, 2006, 177(10): 6758-6768.

[6] V.R. Cicinnati, J. Kang, G.C. Sotiropoulos, et al., Altered chemotactic response of myeloid and plasmacytoid dendritic cells from patients with chronic hepatitis C: Role of alpha interferon. Journal of General Virology, 2008, 89(5): 1243-1253.

[7] F. Gerosa, A. Gobbi, P. Zorzi, et al., The reciprocal interaction of NK cells with plasmacytoid or myeloid dendritic cells profoundly affects innate resistance functions. The Journal of Immunology, 2005, 174(2): 727734.

[8] N. Hagberg, O. Berggren, D. Leonard, et al., IFNproduction by plasmacytoid dendritic cells stimulated with RNA-containing immune complexes is promoted by NK cells via MIP-1 and LFA-1. The Journal of Immunology, 2011, 186(9): 5085-5094.

[9] G. Ferlazzo, B. Morandi, Cross-talks between natural killer cells and distinct subsets of dendritic cells. Frontiers in Immunology, 2014, 5.

[10] G. Alter, M. Altfeld, Mutiny or scrutiny: NK cell modulation of DC function in HIV-1 infection. Trends in Immunology, 2011, 32(5): 219-224.

[11] L.L. Lanier, Natural killer cell receptors and MHC class I interactions. Current Opinion in Immunology, 1997, 9(1): 126-131.

[12] E. Vivier, E. Tomasello, M. Baratin, et al., Functions of natural killer cells. Nature Immunology, 2008, 9(5): 503510.

[13] J.D. Brandstadter, Y. Yang, Natural killer cell responses to viral infection. Journal of Innate Immunity, 2011, 3(3): 274-279.

[14] A. Moretta, E. Marcenaro, S. Parolini, et al., NK cells at the interface between innate and adaptive immunity. Cell Death and Differentiation, 2007, 15(2): 226-233.

[15] D. Pende, R. Castriconi, P. Romagnani, et al., Expression of the DNAM-1 ligands, Nectin-2 (CD112) and poliovirus receptor (CD155), on dendritic cells: relevance for natural killer-dendritic cell interaction. Blood, 2006, 107(5): 2030-2036.

[16] A. Malhotra, A. Shanker, NK cells: Immune cross-talk and therapeutic implications. Immunotherapy, 2011, 3(10): 1143-1166.

[17] D. Vremec, J. Hansen, A. Strasser, et al., Maintaining dendritic cell viability in culture. Molecular immunology, 2015, 63(2): 264-267.

[18] A. Dzionek, Y. Sohma, J. Nagafune, et al., BDCA-2, a novel plasmacytoid dendritic cell-specific type II C-type lectin, mediates antigen capture and is a potent inhibitor of interferon $\alpha / \beta$ induction. Journal of Experimental Medicine, 2001, 194(12): 1823-1834.

[19] A. Dzionek, Y. Inagaki, K. Okawa, et al., Plasmacytoid dendritic cells: from specific surface markers to specific cellular functions1. Human Immunology, 2002, 63(12): 1133-1148.

[20] E. Grage-Griebenow, S. Löseke, M. Kauth, et al., Anti BDCA - 4 (neuropilin - 1) antibody can suppress virus induced IFN-alpha production of plasmacytoid dendritic cells. Immunology \& Cell Biology, 2007, 85(5): 383-390.

[21] S. Elmore, Apoptosis: A review of programmed cell death. Toxicologic Pathology, 2007, 35(4): 495-516.

[22] A. Goc, S.T. Kochuparambil, B. Al-Husein, et al., Simultaneous modulation of the intrinsic and extrinsic pathways by simvastatin in mediating prostate cancer cell apoptosis. BMC Cancer, 2012, 12(1): 409.

[23] R.R. Rosato, J.A. Almenara, Y. Dai, et al., Simultaneous activation of the intrinsic and extrinsic pathways by histone deacetylase (HDAC) inhibitors and tumor necrosis factor-related apoptosis-inducing ligand (TRAIL) synergistically induces mitochondrial damage and apoptosis in human leukemia cells. Molecular Cancer Therapeutics, 2003, 2(12): 1273-1284.

[24] E.M. Carrington, J.G. Zhang, R.M. Sutherland, et al., Prosurvival Bcl-2 family members reveal a distinct apoptotic identity between conventional and plasmacytoid dendritic cells. Proceedings of the National Academy of Sciences, 2015, 112(13): 4044-4049.

Copyright $₫$ Janelle Guy, Emmanuella Sobo, Lechi Nwanegwo, and Kadir Aslan. This is an open-access article distributed under the terms of the Creative Commons Attribution License, which permits unrestricted use, distribution, and reproduction in any medium, provided the original author and source are credited. 